Portland State University

PDXScholar

Electrical and Computer Engineering Faculty

Publications and Presentations

$5-1-1991$

Field-Equation Approximations and the Dynamics of

High-Gain Lasers

Lee W. Casperson

Portland State University

Follow this and additional works at: https://pdxscholar.library.pdx.edu/ece_fac

Part of the Electrical and Computer Engineering Commons

Let us know how access to this document benefits you.

Citation Details

Casperson, L. W. (1991). Field-equation approximations and the dynamics of high-gain lasers. 43 (9), 5057-5067.

This Article is brought to you for free and open access. It has been accepted for inclusion in Electrical and Computer Engineering Faculty Publications and Presentations by an authorized administrator of PDXScholar. Please contact us if we can make this document more accessible: pdxscholar@pdx.edu. 


\title{
Field-equation approximations and the dynamics of high-gain lasers
}

\author{
Lee W. Casperson* \\ Department of Physics, University of Otago, P.O. Box 56, Dunedin, New Zealand
}

(Received 26 September 1990)

\begin{abstract}
Semiclassical models for laser-dynamics studies typically incorporate a derivative approximation that reduces the second-order wave equation for the electric field to a first-order equation. It is shown here that this approximation and further frequency approximations are not necessary and may lead to significant errors for some high-gain laser systems. A more exact analysis also reveals a partial decoupling of the electric and magnetic fields that occurs with fast transient phenomena. These ideas are illustrated in terms of well-known dynamical effects.
\end{abstract}

\section{INTRODUCTION}

One of the most challenging categories of laser behavior includes the transient phenomena that are sometimes obtained in single-mode and multimode lasers. Much theoretical effort has been expended in trying to explain the sometimes wonderfully complex experimental observations, and much experimental effort has been expended in trying to justify the sometimes wonderfully simple theoretical models. One area of emphasis here concerns a particular category of laser instability, but the method of analysis is applicable to a wide range of dynamics problems in lasers and nonlinear optics. The first semiclassical instability to be thoroughly studied is that occurring in the single-mode high-gain xenon laser, ${ }^{1,2}$ and good agreement between the experimental data and a Maxwell-Schrödinger theoretical model is now possible. ${ }^{3}$ Many further examples of interesting laser instabilities have also been discovered, and several reviews of these developments have been published. ${ }^{4-10}$ The models formulated here are also relevant to the simpler problems of loss switching and relaxation oscillations.

One feature that is common to all theoretical studies of laser dynamics is that they inevitably involve several approximations. No aspect of a laser can ever be treated exactly, and one generally adopts the more modest goal of developing models that provide a correct qualitative description of laser behavior while ideally having quantitative errors of at most a few percent. To check that this goal is being achieved, it is necessary, of course, to compare theoretical results with experimental data or at least from time to time to reexamine any dubious approximations. The purpose of this study is to test an approximation that is employed in most studies of laser dynamics. In treating the electromagnetic aspects of light-matter interactions in lasers and other systems, Maxwell's equations are combined to form a second-order wave equation. This wave equation is then reduced to first order with a derivative approximation based on the assumption that the wave envelope varies negligibly within a time of one optical cycle or a distance of one wavelength. With modern high-gain laser media and very short optical pulses, it is worthwhile to explore the possible limitations of this approximation. We consider here the effects of this approximation on the particular semiclassical laser instability mentioned above and on other familiar lossswitching problems.

A general semiclassical model is developed in Sec. II for the dynamics of a laser having arbitrary levels of homogeneous and inhomogeneous line broadening, and a procedure is described for avoiding the derivative approximation in the field equations while adding very little difficulty to the necessary methods. In Sec. III the model is further developed for the special case of a homogeneously broadened laser, and in Sec. IV the implications of the approximation are considered in detail for the familiar semiclassical instability of a homogeneously broadened laser. Avoiding the derivative approximation reveals, for example, that in high-gain wide-band lasers the electric and magnetic fields do not maintain a fixed phase relationship to each other. In Sec. $V$ the model is applied to the familiar problems of giant pulses and relaxation oscillations in a loss-switched laser oscillator.

\section{GENERAL MODEL}

A semiclassical laser model is usually understood to be one in which the atomic or molecular variables are governed by Schrödinger's equation while the electromagnetic fields are solutions of Maxwell's equations. The possible avoidance of a standard derivative approximation of the electromagnetic field equations will be illustrated here for a semiclassical model that governs a broad range of laser dynamical phenomena. The starting point for these considerations is Maxwell's equations in differential form:

$$
\begin{aligned}
& \nabla \times \mathbf{E}=-\frac{\partial \mathbf{B}}{\partial t}, \\
& \nabla \times \mathbf{H}=\frac{\partial \mathbf{D}}{\partial t}+\mathbf{J}
\end{aligned}
$$

Before proceeding to a more exact treatment, the usual approximate incorporation of these equations in the model will be briefly reviewed. This process will also provide a starting point for the following more exact analysis.

It will be assumed here that the laser medium has scalar permittivity, permeability, and conductivity and that the electromagnetic field is a plane wave polarized in the 
$x$ direction and propagating in the $z$ direction. Thus, the vector field equations reduce to the scalar equations

$$
\begin{aligned}
& \frac{\partial E_{x}(z, t)}{\partial z}=-\frac{\partial B_{y}(z, t)}{\partial t} \\
& \frac{\partial H_{y}(z, t)}{\partial z}=-\frac{\partial D_{x}(z, t)}{\partial t}-J_{x}(z, t) .
\end{aligned}
$$

From this point the polarization subscripts will be dropped, and the following constitutive relations will be adopted:

$$
\begin{aligned}
& D(z, t)=\epsilon_{0} E(z, t)+P_{t}(z, t)=\epsilon_{1} E(z, t)+P(z, t), \\
& B(z, t)=\mu_{0} H(z, t)+M_{t}(z, t)=\mu_{1} H(z, t)+M(z, t), \\
& J(z, t)=\sigma E(z, t)
\end{aligned}
$$

where $P_{t}(z, t)$ and $M_{t}(z, t)$ are the total polarization and magnetization, respectively. The permittivity and permeability independent of the polarization $P(z, t)$ and the magnetization $M(z, t)$ of the lasing atoms or molecules are represented by $\epsilon_{1}$ and $\mu_{1}$, respectively, and for the case of interest here the extra magnetization $M(z, t)$ is equal to zero. With these constitutive relations Eqs. (3) and (4) reduce to

$$
\begin{aligned}
& \frac{\partial E(z, t)}{\partial z}=-\mu_{1} \frac{\partial H(z, t)}{\partial t}, \\
& \frac{\partial H(z, t)}{\partial z}=-\epsilon_{1} \frac{\partial E(z, t)}{\partial t}-\frac{\partial P(z, t)}{\partial t}-\sigma E(z, t) .
\end{aligned}
$$

Maxwell's equations are usually combined immediately into a second-order wave equation. If one differentiates Eq. (8) with respect to $z$ and Eq. (9) with respect to $t$, one obtains the familiar result

$$
\begin{aligned}
& \frac{\partial^{2} E(z, t)}{\partial z^{2}}-\mu_{1} \sigma \frac{\partial E(z, t)}{\partial t}-\mu_{1} \epsilon_{1} \frac{\partial^{2} E(z, t)}{\partial t^{2}} \\
&=\mu_{1} \frac{\partial^{2} P(z, t)}{\partial t^{2}} .
\end{aligned}
$$

In a semiclassical model for a general inhomogeneously broadened laser, the polarization driving this equation can be related back to the off-diagonal density matrix elements by

$P(z, t)=\int_{0}^{\infty} \int_{-\infty}^{\infty} \mu \rho_{a b}\left(v, \omega_{\alpha}, z, t\right) d v d \omega_{\alpha}+$ c.c. ,

where $v$ is the velocity and $\omega_{\alpha}$ is the center frequency of the laser transition for members of an atomic or molecular class $\alpha, \mu$ is the dipole moment of the transition, and the notation c.c. means the complex conjugate of the preceding terms. Taken together with the density matrix form of Schrödinger's equation, Eqs. (10) and (11) provide a complete set from which the time and space dependences of the electric field and of the atomic or molecular parameters can be determined, subject to the boundary conditions at the resonator mirrors.

The simplest dynamical solutions for the model that has just been described are those that apply to uniformly pumped one-directional ring lasers, in which both the electric field and the polarization are traveling waves. If the gain and loss in a ring laser can be considered to be uniformly distributed over a completely filled cavity, then the rapid time and space variations in the model can be factored out by means of the substitutions

$E(z, t)=\frac{1}{2} E^{\prime}(t) \exp (i k z-i \omega t)+$ c.c. ,

$\rho_{a b}\left(v, \omega_{\alpha}, z, t\right)=P^{\prime}\left(v, \omega_{\alpha}, t\right) \exp (i k z-i \omega t) / 2 \mu$.

Equations (10) to (13) may be combined to obtain the new spatially independent wave equation for the complex field amplitude $E^{\prime}(t)$ :

$$
\begin{aligned}
\frac{d^{2} E^{\prime}(t)}{d t^{2}} & +\left(\frac{\sigma}{\epsilon_{1}}-2 i \omega\right) \frac{d E^{\prime}(t)}{d t}-\left[\omega^{2}-\Omega^{2}+i \frac{\sigma}{\epsilon_{1}} \omega\right] E^{\prime}(t) \\
& =-\frac{1}{\epsilon_{1}}\left[\frac{\partial^{2}}{\partial t^{2}} \int_{0}^{\infty} \int_{-\infty}^{\infty} P^{\prime}\left(v, \omega_{\alpha}, t\right) d v d \omega_{\alpha}-2 i \omega \frac{\partial}{\partial t} \int_{0}^{\infty} \int_{-\infty}^{\infty} P^{\prime}\left(v, \omega_{\alpha}, t\right) d v d \omega_{\alpha}-\omega^{2} \int_{0}^{\infty} \int_{-\infty}^{\infty} P^{\prime}\left(v, \omega_{\alpha}, t\right) d v d \omega_{\alpha}\right]
\end{aligned}
$$

where the new frequency $\Omega=k\left(\mu_{1} \epsilon_{1}\right)^{-1 / 2}$ has been introduced, and the conductivity $\sigma$ is meant to represent all cavity losses. It is now usual to drop some of the higher derivative terms by arguing that the field and polarization envelopes vary negligibly in an optical cycle and that the losses per optical cycle are also negligible. The terms that remain are

$$
\begin{aligned}
\frac{d E^{\prime}(t)}{d t}= & -\gamma_{c} E^{\prime}(t)+i \frac{\left(\omega^{2}-\Omega^{2}\right)}{2 \omega} E^{\prime}(t) \\
& +i \frac{\omega}{2 \varepsilon_{1}} \int_{0}^{\infty} \int_{-\infty}^{\infty} P^{\prime}\left(v, \omega_{\alpha}, t\right) d v d \omega_{\alpha},
\end{aligned}
$$

where the losses are represented by the field decay rate $\gamma_{c}=\sigma / 2 \epsilon_{1}$. The frequency $\Omega$ can now be recognized as the nondispersed cavity resonance frequency, i.e., the lasing frequency that would result if the dispersion or real part of the polarization $P^{\prime}\left(v, \omega_{\alpha}, t\right)$ were equal to zero. A major purpose of this study is to examine the validity and possible limitations of the derivative approximation that has just been employed in reducing Eq. (14) to Eq. (15).

Further frequency approximations are also commonly introduced. If the lasing frequency $\omega$ is close to the nondispersed frequency $\Omega$, then the term $\left(\omega^{2}-\Omega^{2}\right) / 2 \omega$ can be approximated by $\omega-\Omega$, and if the lasing frequency is 
also close to a characteristic frequency of the laser transition $\omega_{0}$, then the $\omega$ multiplying the polarization integrals may be replaced by $\omega_{0}$. With these approximations, Eq. (15) reduces to

$$
\begin{aligned}
\frac{d E^{\prime}(t)}{d t}= & -\gamma_{c} E^{\prime}(t)+i(\omega-\Omega) E^{\prime}(t) \\
& +i \frac{\omega_{0}}{2 \epsilon_{1}} \int_{0}^{\infty} \int_{-\infty}^{\infty} P^{\prime}\left(v, \omega_{\alpha}, t\right) d v d \omega_{\alpha} .
\end{aligned}
$$

This result appears as Eq. (12) in Ref. 3 and in other forms in most semiclassical laser models. The limitations on the validity of the frequency approximations for steady-state laser behavior have recently been considered in detail, ${ }^{11}$ and it will be shown below that these approximations can also readily be avoided in laser-dynamics studies.

Next, it is helpful to separate the field and polarization into their real and imaginary parts in the forms

$$
E^{\prime}(t)=E_{r}(t)+i E_{i}(t)
$$

and

$$
P^{\prime}\left(v, \omega_{\alpha}, t\right)=P_{r}\left(v, \omega_{\alpha}, t\right)+i P_{i}\left(v, \omega_{\alpha}, t\right) .
$$

With these substitutions a useful semiclassical ring laser model is ${ }^{3}$

$$
\begin{aligned}
& \frac{\partial P_{r}\left(v, \omega_{\alpha}, t\right)}{\partial t}=-\left(\omega-\omega_{\alpha}-k v\right) P_{i}\left(v, \omega_{\alpha}, t\right)-\gamma P_{r}\left(v, \omega_{\alpha}, t\right)+\frac{\mu^{2}}{\hbar} E_{i}(t) D\left(v, \omega_{\alpha}, t\right) \\
& \frac{\partial P_{i}\left(v, \omega_{\alpha}, t\right)}{\partial t}=\left(\omega-\omega_{\alpha}-k v\right) P_{r}\left(v, \omega_{\alpha}, t\right)-\gamma P_{i}\left(v, \omega_{\alpha}, t\right)-\frac{\mu^{2}}{\hbar} E_{r}(t) D\left(v, \omega_{\alpha}, t\right) \\
& \frac{\partial D\left(v, \omega_{\alpha}, t\right)}{\partial t}= \lambda_{a}\left(v, \omega_{\alpha}, t\right)-\lambda_{b}\left(v, \omega_{\alpha}, t\right)-\frac{\gamma_{a}+\gamma_{a b}+\gamma_{b}}{2} D\left(v, \omega_{\alpha}, t\right)-\frac{\gamma_{a}+\gamma_{a b}-\gamma_{b}}{2} M\left(v, \omega_{\alpha}, t\right) \\
&+\frac{1}{\hbar}\left[E_{r}(t) P_{i}\left(v, \omega_{\alpha}, t\right)-E_{i}(t) P_{r}\left(v, \omega_{\alpha}, t\right)\right] \\
& \frac{\partial M\left(v, \omega_{\alpha}, t\right)}{\partial t}= \lambda_{a}\left(v, \omega_{\alpha}, t\right)+\lambda_{b}\left(v, \omega_{\alpha}, t\right)-\frac{\gamma_{a}-\gamma_{a b}+\gamma_{b}}{2} D\left(v, \omega_{\alpha}, t\right)-\frac{\gamma_{a}-\gamma_{a b}+\gamma_{b}}{2} M\left(v, \omega_{\alpha}, t\right), \\
& \frac{d E_{r}(t)}{d t}=-\gamma_{c} E_{r}(t)-(\omega-\Omega) E_{i}(t)-\frac{\omega_{0}}{2 \epsilon_{1}} \int_{0}^{\infty} \int_{-\infty}^{\infty} P_{i}\left(v, \omega_{\alpha}, t\right) d v d \omega_{\alpha}, \\
& \frac{d E_{i}(t)}{d t}=-\gamma_{c} E_{i}(t)+(\omega-\Omega) E_{r}(t)+\frac{\omega_{0}}{2 \epsilon_{1}} \int_{0}^{\infty} \int_{-\infty}^{\infty} P_{r}\left(v, \omega_{\alpha}, t\right) d v d \omega_{\alpha},
\end{aligned}
$$

where the subscripts $a$ and $b$ denote the upper and lower laser levels, respectively; $\gamma_{a}$ and $\gamma_{b}$ are the total decay rates for these levels; $\gamma_{a b}$ is the rate of direct decays from level $a$ to level $b ; \gamma$ is the decay rate for the off-diagonal elements; $\lambda_{a}\left(v, \omega_{\alpha}, t\right)$ and $\lambda_{b}\left(v, \omega_{\alpha}, t\right)$ are the pumping rates; and the population difference

$$
D\left(v, \omega_{\alpha}, t\right)=\rho_{a a}\left(v, \omega_{\alpha}, t\right)-\rho_{b b}\left(v, \omega_{\alpha}, t\right)
$$

and sum

$$
M\left(v, \omega_{\alpha}, t\right)=\rho_{a a}\left(v, \omega_{\alpha}, t\right)+\rho_{b b}\left(v, \omega_{\alpha}, t\right)
$$

have also been introduced. For brevity, spectral cross relaxation is omitted.

Equations (17) to (22) summarize an established model for spontaneous coherent pulsations in ring laser oscillators, and these equations are similar to those employed in other ring laser dynamical models. Such models are generally formulated as a set of coupled first-order differential equations, and they are applicable to a wide range of transient phenomena such as instabilities, the giant pulsations arising from loss switching, and damped relaxation oscillations. Our purpose here is to test whether the derivative approximation inherent in Eqs.
(21) and (22) might be introducing significant errors in the predicted laser behavior. To answer this question, one may set up a similar model which includes neither the derivative approximation nor the frequency approximation described above.

The first possibility that one might consider for removing the derivative approximation is simply to replace Eqs. (21) and (22) with the real and imaginary parts of Eq. (14). However, this equation has second-order derivatives in both the field and polarization variables, and numerical solutions are not straightforward. We find that it is more efficient and more informative to avoid forming the second-order wave equation in the first place. Thus, one can instead work directly from Maxwell's equations and retain the significance that the variables correspond directly to the electric and magnetic fields. As a first step, one must also factor out the rapid time and space variations in the magnetic field, and a useful substitution is

$H(z, t)=\frac{1}{2}\left(\epsilon_{1} / \mu_{1}\right)^{1 / 2} H^{\prime}(t) \exp (i k z-i \omega t)+$ c.c.

If Eqs. (11) to (13) and (23) are substituted into Eqs. (8) and (9), one obtains 


$$
\begin{aligned}
\frac{d E^{\prime}(t)}{d t}= & -2 \gamma_{c} E^{\prime}(t)+i\left[\omega E^{\prime}(t)-\Omega H^{\prime}(t)\right] \\
& -\frac{1}{\epsilon_{1}} \int_{0}^{\infty} \int_{-\infty}^{\infty} \frac{\partial P^{\prime}\left(v, \omega_{\alpha}, t\right)}{\partial t} d v d \omega_{\alpha} \\
& +i \frac{\omega}{\epsilon_{1}} \int_{0}^{\infty} \int_{-\infty}^{\infty} P^{\prime}\left(v, \omega_{\alpha}, t\right) d v d \omega_{\alpha} \\
\frac{d H^{\prime}(t)}{d t}= & i\left[\omega H^{\prime}(t)-\Omega E^{\prime}(t)\right] .
\end{aligned}
$$

Equations (24) and (25) may be separated into their real and imaginary parts:

$$
\begin{aligned}
\frac{d E_{r}(t)}{d t}= & -2 \gamma_{c} E_{r}(t)-\left[\omega E_{i}(t)-\Omega H_{i}(t)\right] \\
& -\frac{1}{\epsilon_{1}} \int_{0}^{\infty} \int_{-\infty}^{\infty} \frac{\partial P_{r}\left(v, \omega_{\alpha}, t\right)}{\partial t} d v d \omega_{\alpha} \\
& -\frac{\omega}{\epsilon_{1}} \int_{0}^{\infty} \int_{-\infty}^{\infty} P_{i}\left(v, \omega_{\alpha}, t\right) d v d \omega_{\alpha} \\
\frac{d E_{i}(t)}{d t}= & -2 \gamma_{c} E_{i}(t)+\left[\omega E_{r}(t)-\Omega H_{r}(t)\right] \\
& -\frac{1}{\epsilon_{1}} \int_{0}^{\infty} \int_{-\infty}^{\infty} \frac{\partial P_{i}\left(v, \omega_{\alpha}, t\right)}{\partial t} d v d \omega_{\alpha} \\
& +\frac{\omega}{\epsilon_{1}} \int_{0}^{\infty} \int_{-\infty}^{\infty} P_{r}\left(v, \omega_{c}, t\right) d v d \omega_{\alpha} \\
\frac{d H_{r}(t)}{d t}= & -\left[\omega H_{i}(t)-\Omega E_{i}(t)\right] \\
\frac{d H_{i}(t)}{d t}= & {\left[\omega H_{r}(t)-\Omega E_{r}(t)\right] . }
\end{aligned}
$$

The polarization derivatives in Eqs. (26) and (27) may be eliminated by means of Eqs. (17) and (18), and then the model consisting of Eqs. (17) to (20) and (26) to (29) may be applied to a variety of instability and other dynamical problems concerning laser oscillators. It should be emphasized that this revised model entirely avoids the derivative and frequency approximations inherent in other laser-dynamics formalisms without adding substantially to the difficulty of the numerical solutions.

\section{HOMOGENEOUS BROADENING}

The purpose of the foregoing analysis has been to establish a formalism for treating the evolution of very fast transients in a general class of mixed-broadened ring laser oscillators. However, it is not necessary to solve the most general laser configurations to obtain an estimate of the implications of the more exact model. In this discussion we will focus on only the simplest special case. Following the simplifications developed in an earlier study, a normalized form of Eqs. (17) to (22) can be reduced to ${ }^{12}$

$$
\begin{gathered}
\frac{\partial P_{r}(V, t)}{\partial t}=-\gamma\left[P_{r}(V, t)+(y-V) P_{i}(V, t)\right. \\
\left.-A_{i}(t) D(V, t)\right],
\end{gathered}
$$

$$
\begin{aligned}
& \frac{\partial P_{i}(V, t)}{\partial t}=-\gamma\left[P_{i}(V, t)-(y-V) P_{r}(V, t)\right. \\
& \left.+A_{r}(t) D(V, t)\right], \\
& \frac{\partial D(V, t)}{\partial t}=-\gamma_{d}\left[D(V, t)-D_{0}(V, t)\right. \\
& \left.-A_{r}(t) P_{i}(V, t)+A_{i}(t) P_{r}(V, t)\right], \\
& \frac{d A_{r}(t)}{d t}=-\gamma_{c}\left(A_{r}(t)+\delta\left(y-y_{0}\right) A_{i}(t)\right. \\
& \left.+\int_{-\infty}^{\infty} P_{i}(V, t) d V\right], \\
& \frac{d A_{i}(t)}{d t}=-\gamma_{c}\left[A_{i}(t)-\delta\left(y-y_{0}\right) A_{r}(t)\right. \\
& \left.-\int_{-\infty}^{\infty} P_{r}(V, t) d V\right]
\end{aligned}
$$

where all of the atoms now have the same center frequency $\left(\omega_{\alpha}=0\right)$, and the spontaneous decay rates are such that the equation for the population sum $M$ drops out. The pump rate is represented by $D_{0}(V, t)$; the parameter $y=\left(\omega-\omega_{0}\right) / \gamma$ is the normalized lasing frequency; $y_{0}=\left(\Omega-\omega_{0}\right) / \gamma$ is the normalized cavity frequency; $V=k v / \gamma$ is the normalized velocity; $\delta=\gamma / \gamma_{c}$ is a dimensionless decay rate ratio; and $A_{r}(t)$ and $A_{i}(t)$ are, respectively, the normalized real and imaginary components of the electric field.

Equations (30) to (34) are a conventional model for the dynamics of an inhomogeneously broadened laser, and Eqs. (33) and (34) have been obtained using the standard approximations discussed above. If these approximations are not to be employed, it follows from Eqs. (26) to (29) that the field equations should be replaced by

$$
\begin{aligned}
& \frac{d A_{r}(t)}{d t}=-\gamma_{c} \mid 2 A_{r}(t) \\
&+\delta\left[\left(y+z_{0}\right) A_{i}(t)-\left(y_{0}+z_{0}\right) B_{i}(t)\right] \\
&+\frac{2}{\gamma z_{0}} \int_{-\infty}^{\infty} \frac{\partial P_{r}(V, t)}{\partial t} d V \\
&\left.+\frac{2\left(y+z_{0}\right)}{z_{0}} \int_{-\infty}^{\infty} P_{i}(V, t) d V\right], \\
& \frac{d A_{i}(t)}{d t}=-\gamma_{c}\left[\begin{array}{l}
2 \\
\end{array}\right.-\delta\left[\left(y+z_{0}\right) A_{r}(t)-\left(y_{0}+z_{0}\right) B_{r}(t)\right] \\
&+\frac{2}{\gamma z_{0}} \int_{-\infty}^{\infty} \frac{\partial P_{i}(V, t)}{\partial t} d V \\
&\left.-\frac{2\left(y+z_{0}\right)}{z_{0}} \int_{-\infty}^{\infty} P_{r}(V, t) d V\right], \\
& \frac{d B_{r}(t)}{d t}=-\gamma_{c} \delta\left[\left(y+z_{0}\right) B_{i}(t)-\left(y_{0}+z_{0}\right) A_{i}(t)\right],
\end{aligned}
$$


$\frac{d B_{i}(t)}{d t}=\gamma_{c} \delta\left[\left(y+z_{0}\right) B_{r}(t)-\left(y_{0}+z_{0}\right) A_{r}(t)\right]$,

where $B_{r}(t)$ and $B_{i}(t)$ are, respectively, the real and imaginary components of the magnetic field with the same normalization as used for the electric field, and $z_{0}=\omega_{0} / \gamma$ is the normalized center frequency of the transition. As noted previously, the polarization derivatives in Eqs. (35) and (36) can be eliminated by means of Eqs. (30) and (31). The resulting equation set can then be integrated in the usual way, using, for example, a Runge-Kutta method for the time derivatives and Simpson's rule for the velocity integrals. $^{2}$

Equations (30) to (38) represent both the relatively exact and the derivative-approximate forms of the laserdynamics equations for an amplifying medium with an arbitrary level of Doppler broadening. For the laser instability of interest here, it has been shown previously that in the limit of strong Doppler broadening even the first field derivatives are not essential for good qualitative accuracy. ${ }^{12}$ Hence, for present purposes attention will be restricted to the opposite limit of homogeneous broadening. With $V=0$ and an obvious redefinition of variables, Eqs. (30) to (38) reduce to

$$
\begin{aligned}
& \frac{d P_{r}(t)}{d t}=-\gamma\left[P_{r}(t)+y P_{i}(t)-A_{i}(t) D(t)\right] \\
& \frac{d P_{i}(t)}{d t}=-\gamma\left[P_{i}(t)-y P_{r}(t)+A_{r}(t) D(t)\right] \\
& \frac{d D(t)}{d t}=-\gamma_{d}\left[D(t)-D_{0}(t)-A_{r}(t) P_{i}(t)+A_{i}(t) P_{r}(t)\right]
\end{aligned}
$$

$$
\begin{aligned}
& \frac{d A_{r}(t)}{d t}=-\gamma_{c}\left[A_{r}(t)+\delta\left(y-y_{0}\right) A_{i}(t)+P_{i}(t)\right] \\
& \frac{d A_{i}(t)}{d t}=-\gamma_{c}\left[A_{i}(t)-\delta\left(y-y_{0}\right) A_{r}(t)-P_{r}(t)\right]
\end{aligned}
$$

$$
\begin{aligned}
\frac{d A_{r}(t)}{d t}=-\gamma_{c}[ & 2 A_{r}(t) \\
& +\delta\left[\left(y+z_{0}\right) A_{i}(t)-\left(y_{0}+z_{0}\right) B_{i}(t)\right] \\
& \left.+\frac{2}{\gamma z_{0}} \frac{d P_{r}(t)}{d t}+\frac{2\left(y+z_{0}\right)}{z_{0}} P_{i}(t)\right],
\end{aligned}
$$

$$
\begin{aligned}
\frac{d A_{i}(t)}{d t}=-\gamma_{c}( & 2 A_{i}(t) \\
& -\delta\left[\left(y+z_{0}\right) A_{r}(t)-\left(y_{0}+z_{0}\right) B_{r}(t)\right] \\
& \left.+\frac{2}{\gamma z_{0}} \frac{d P_{i}(t)}{d t}-\frac{2\left(y+z_{0}\right)}{z_{0}} P_{r}(t)\right],
\end{aligned}
$$

$\frac{d B_{r}(t)}{d t}=-\gamma_{c} \delta\left[\left(y+z_{0}\right) B_{i}(t)-\left(y_{0}+z_{0}\right) A_{i}(t)\right]$,

$\frac{d B_{i}(t)}{d t}=\gamma_{c} \delta\left[\left(y+z_{0}\right) B_{r}(t)-\left(y_{0}+z_{0}\right) A_{r}(t)\right]$.
The set including Eqs. (39) to (43) is a standard model for the dynamics of a homogeneously broadened laser with arbitrary detuning. Replacing Eqs. (42) and (43) in the model with Eqs. (44) to (47) avoids the derivative and frequency approximations.

As a final simplification, the laser cavity mode is tuned to the center of the atomic transition $\left(y_{0}=0\right)$ and the arbitrary oscillation frequency is also placed at line center $(y=0)$. Then Eqs. (39) to (47) reduce to

$$
\begin{aligned}
& \frac{d P_{r}(t)}{d t}=-\gamma\left[P_{r}(t)-A_{i}(t) D(t)\right], \\
& \frac{d P_{i}(t)}{d t}=-\gamma\left[P_{i}(t)+A_{r}(t) D(t)\right], \\
& \frac{d D(t)}{d t}=-\gamma_{d}\left[D(t)-D_{0}(t)-A_{r}(t) P_{i}(t)\right. \\
& \left.+A_{i}(t) P_{r}(t)\right], \\
& \frac{d A_{r}(t)}{d t}=-\gamma_{c}\left[A_{r}(t)+P_{i}(t)\right], \\
& \frac{d A_{i}(t)}{d t}=-\gamma_{c}\left[A_{i}(t)-P_{r}(t)\right], \\
& \frac{d A_{r}(t)}{d t}=-\gamma_{c}\left[2 A_{r}(t)+\delta z_{0}\left[A_{i}(t)-B_{i}(t)\right]\right. \\
& \left.+\frac{2}{\gamma z_{0}} \frac{d P_{r}(t)}{d t}+2 P_{i}(t)\right] \\
& \frac{d A_{i}(t)}{d t}=-\gamma_{c}\left[2 A_{i}(t)-\delta z_{0}\left[A_{r}(t)-B_{r}(t)\right]\right. \\
& \left.+\frac{2}{\gamma z_{0}} \frac{d P_{i}(t)}{d t}-2 P_{r}(t)\right] \\
& \frac{d B_{r}(t)}{d t}=-\gamma_{c} \delta z_{0}\left[B_{i}(t)-A_{i}(t)\right], \\
& \frac{d B_{i}(t)}{d t}=\gamma_{c} \delta z_{0}\left[B_{r}(t)-A_{r}(t)\right] .
\end{aligned}
$$

The approximate set consisting of Eqs. (48) to (52) actually simplifies a little further since for an appropriate choice of phase $A_{i}(t)$ and $P_{r}(t)$ can be set equal to zero. As shown by Haken, the resulting three equations are mathematically equivalent to the Lorenz equations of hydrodynamics. ${ }^{13}$ The corresponding set with the more exact treatment of the fields consists of Eqs. (48) to (50) and (53) to (56). With Eqs. (48) and (49) the polarization derivatives may be eliminated from Eqs. (53) and (54), and the results are

$$
\begin{aligned}
& \frac{d A_{r}(t)}{d t}=-\gamma_{c}\left[2 A_{r}(t)+\delta z_{0}\left[A_{i}(t)-B_{i}(t)\right]\right. \\
&\left.-\frac{2}{z_{0}}\left[P_{r}(t)-A_{i}(t) D(t)\right]+2 P_{i}(t)\right],
\end{aligned}
$$




$$
\begin{aligned}
\frac{d A_{i}(t)}{d t}=-\gamma_{c}\left[\begin{array}{l}
2 A_{i}(t)-\delta z_{0}\left[A_{r}(t)-B_{r}(t)\right] \\
\end{array}\right. & \left.-\frac{2}{z_{0}}\left[P_{i}(t)+A_{r}(t) D(t)\right]-2 P_{r}(t)\right],
\end{aligned}
$$

Thus, while the more accurate equation set consisting of Eqs. (48) to (50) and (55) to (58) is larger, the parameters involved are basically the same and the equations have the same structure as the conventional three-equation set.

For laser instability studies it is convenient to express the laser behavior in terms of the threshold parameter $r$, which is the ratio of the constant pumping rate $D_{0}$ to its value at the lasing threshold. One readily finds from the unsaturated steady-state solutions that $D_{0}$ has the value unity at the lasing threshold for both of the instability models contained in Eqs. (48) to (52) and (55) to (58). Therefore, the parameter $D_{0}(t)$ may simply be replaced by the threshold parameter $r$ in the models.

The preceding analysis provides a formalism for calculating the instantaneous electric and magnetic fields in a laser oscillator. Next, it is necessary to identify for display some specific quantities of practical interest. For this purpose a version of Poynting's theorem can be constructed by multiplying Eq. (8) by $H(z, t)$ and Eq. (9) by $E(z, t)$ and then adding to obtain

$$
\begin{aligned}
\frac{\partial}{\partial z}[E(z, t) H(z, t)]= & -\frac{\epsilon_{1}}{2} \frac{\partial E^{2}(z, t)}{\partial t}-\frac{\mu_{1}}{2} \frac{\partial H^{2}(z, t)}{\partial t} \\
& -E(z, t) \frac{\partial P(z, t)}{\partial t}-\sigma E^{2}(z, t)
\end{aligned}
$$

With the field definitions given in Eqs. (12) and (23), one can identify Poynting's vector:

$$
\begin{aligned}
S(z, t) & =E(z, t) H(z, t) \\
& =\left[\frac{1}{2} E^{\prime}(t) \exp (i k z-i \omega t)+\text { c.c. }\right]\left[\frac{1}{2}\left(\epsilon_{1} / \mu_{1}\right)^{1 / 2} H^{\prime}(t) \exp (i k z-i \omega t)+\text { c.c. }\right] \\
& =\frac{1}{4}\left(\epsilon_{1} / \mu_{1}\right)^{1 / 2}\left[E^{\prime}(t) H^{\prime *}(t)+E^{\prime}(t) H^{\prime}(t) \exp (2 i k z-2 i \omega t)+\text { c.c. }\right],
\end{aligned}
$$

as well as energy densities associated with the electric and magnetic fields

$$
\begin{aligned}
u_{e}(z, t) & =\left(\epsilon_{1} / 2\right) E^{2}(z, t) \\
& =\left(\epsilon_{1} / 8\right)\left[E^{\prime}(t) E^{\prime *}(t)+E^{\prime}(t) E^{\prime}(t) \exp (2 i k z-2 i \omega t)+\text { c.c. }\right], \\
u_{m}(z, t) & =\left(\mu_{1} / 2\right) H^{2}(z, t) \\
& =\left(\epsilon_{1} / 8\right)\left[H^{\prime}(t) H^{\prime *}(t)+H^{\prime}(t) H^{\prime}(t) \exp (2 i k z-2 i \omega t)+\text { c.c. }\right] .
\end{aligned}
$$

It should be noted that $u_{e}(z, t)$ in Eq. $(61)$ is not the total electric energy density, because it explicitly excludes the term in Eq. (59) involving the electric polarization $P(z, t)$ associated with the lasing atoms or molecules. Only when all polarization terms are directly proportional to the corresponding instantaneous fields do the total energy densities have a simple interpretation.

Like the fields themselves, the energy-related quantities in Eqs. (60) to (62) are a bit too complicated to provide a convenient representation of the laser behavior. However, averaging over a distance of one half wavelength eliminates the complex exponentials and leads to the simpler formulas

$$
\begin{aligned}
I^{\prime}(t) & =2\left(\mu_{1} / \epsilon_{1}\right)^{1 / 2}\langle S(z, t)\rangle \\
& =\frac{1}{2}\left[E^{\prime}(t) H^{\prime *}(t)+E^{\prime *}(t) H^{\prime}(t)\right] \\
& =\frac{1}{2}\left\{\left[E_{r}(t)+i E_{i}(t)\right]\left[H_{r}(t)-i H_{i}(t)\right]+\left[E_{r}(t)-i E_{i}(t)\right]\left[H_{r}(t)+i H_{i}(t)\right]\right\} \\
& =E_{r}(t) H_{r}(t)+E_{i}(t) H_{i}(t), \\
U_{e}^{\prime}(t) & =\left(4 / \epsilon_{1}\right)\left\langle u_{e}(z, t)\right\rangle=E^{\prime}(t) E^{\prime *}(t)=E_{r}^{2}(t)+E_{i}^{2}(t), \\
U_{m}^{\prime}(t) & =\left(4 / \epsilon_{1}\right)\left\langle u_{m}(z, t)\right\rangle=H^{\prime}(t) H^{\prime *}(t)=H_{r}^{2}(t)+H_{i}^{2}(t) .
\end{aligned}
$$

In terms of the normalized field variables, Eqs. (63) to (65) are

$$
\begin{aligned}
& I(t)=A_{r}(t) B_{r}(t)+A_{i}(t) B_{i}(t), \\
& U_{e}(t)=A_{r}^{2}(t)+A_{i}^{2}(t), \\
& U_{m}(t)=B_{r}^{2}(t)+B_{i}^{2}(t) .
\end{aligned}
$$

For ordinary low-frequency plane-wave applications, the normalized electric field amplitudes $A_{r}(t)$ and $A_{i}(t)$ are also almost equal to the magnetic field amplitudes $B_{r}(t)$ and $B_{i}(t)$. In that limit the three energy measures given in Eqs. (66) to (68) are also almost equal. However, for the very high gain systems of interest here, it will be found that the electric and magnetic energies may differ 
substantially.

The functions given in Eqs. (66) to (68) focus, in effect, on the amplitude characteristics of the electromagnetic fields. The phase characteristics are also of interest. One convenient way to explore the phase properties of the rapidly varying laser fields is in terms of their instantaneous frequency. ${ }^{14}$ This frequency is defined as the time derivative of the total phase of the field. Thus, for the electric field discussed above, the time-dependent frequency can be written

$$
\omega_{e}(t)=\omega-\frac{d}{d t}\left\{\tan ^{-1}\left[A_{i}(t) / A_{r}(t)\right]\right\} .
$$

In this study the electric and magnetic fields are partially decoupled, and it is possible for the instantaneous frequency of the electric field to be different from the instantaneous frequency of the magnetic field, which is given by

$$
\omega_{m}(t)=\omega-\frac{d}{d t}\left\{\tan ^{-1}\left[B_{i}(t) / B_{r}(t)\right]\right\} .
$$

These formulas are evaluated in the following section for a spontaneously pulsing laser oscillator.

\section{SPONTANEOUS PULSATIONS}

A range of numerical studies has been carried out to examine the implications of the derivative approximation for spontaneous pulsations in a laser oscillator. A typical set of intensity wave forms for a homogeneously broadened laser is plotted in Fig. 1. The computations in this case are based on the model given in Eqs. (48) to (50) and (55) to (58), and the intensity is defined in Eq. (66). The solutions are obtained using a second-order RungeKutta method. The laser parameters used in these calculations include the decay rate ratios $\delta=\gamma / \gamma_{c}=0.3$ and
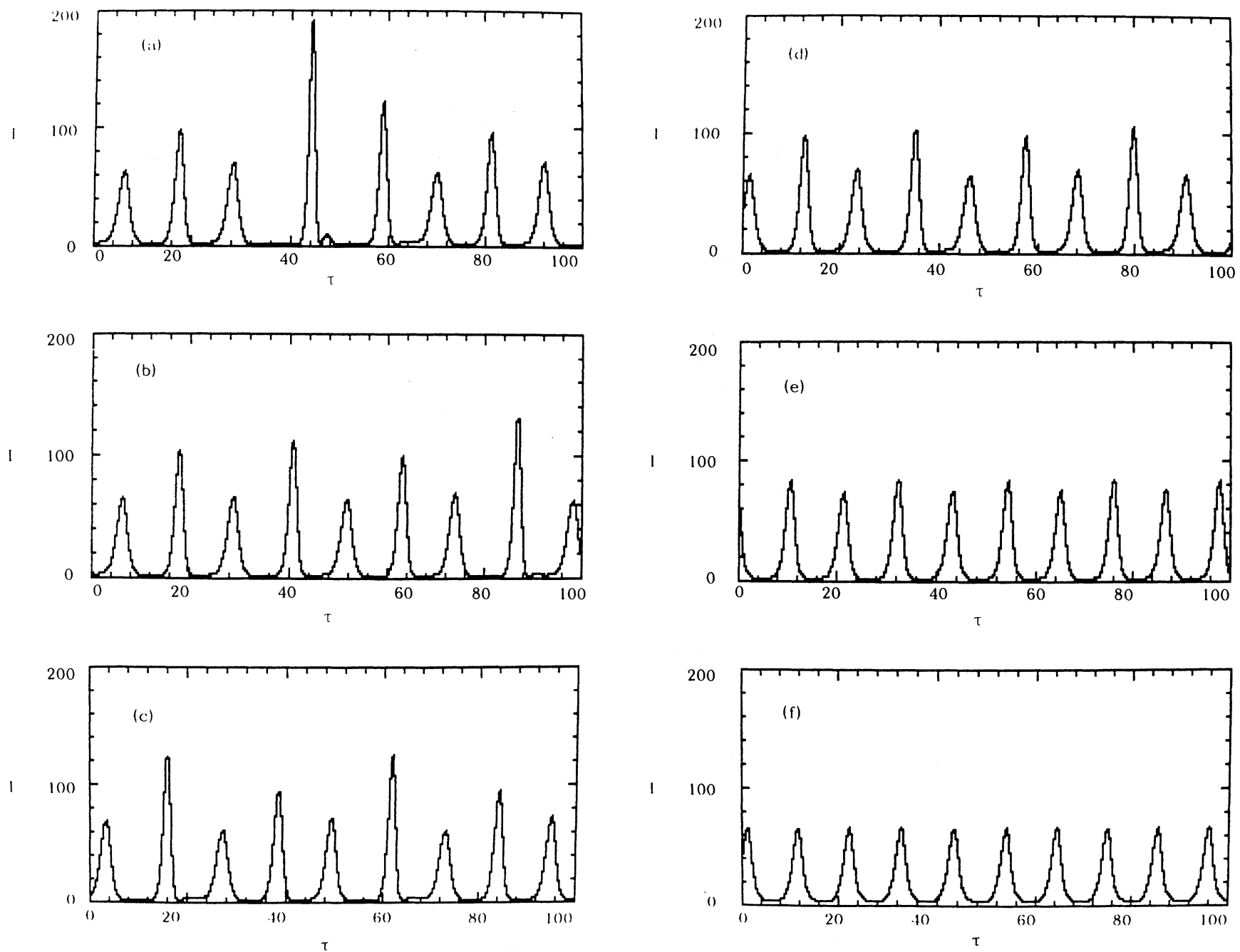

FIG. 1. Spontaneous pulsation intensity wave forms for a laser having the decay rate ratios $\delta=\gamma / \gamma_{c}=0.3$ and $\rho=\gamma_{d} / \gamma=0.2$ and the threshold parameter $r=20$. The normalized center frequencies $z_{0}=\omega_{0} / \gamma$ for these plots are (a) $\infty$, (b) 100 , (c) 50 , (d) 20 , (e) 10 , and (f) 5. It is clear from these plots that decreasing frequencies (or increasing homogeneous linewidths) for this range of operation cause increasing stabilization of the pulsations and greater disagreement with the Lorenz-equivalent model. 
$\rho=\gamma_{d} / \gamma=0.2$, and the threshold parameter is $r=20$. The intensity is plotted against the normalized time $\tau=\gamma_{c} t$ for several values of the normalized center frequency $z_{0}=\omega_{0} / \gamma$.

The curve in Fig. 1(a) is obtained for the limit $z_{0}=\infty$, and in this limit the field equations given in Eqs. (55) to (58) reduce to Eqs. (51) and (52). This may be seen by considering first Eqs. (55) and (56). In order for the magnetic-field derivatives in these equations to remain finite for large values of $z_{0}$, the electric and magnetic fields must approach equality. Thus, the terms in Eqs. (57) and (58) which are products of the small differences between the electric and magnetic fields and the large frequency $z_{0}$ may be replaced by the corresponding electric-field derivatives. Then Eqs. (57) and (58) reduce easily to Eqs. (51) and (52), which are the basis of the familiar Lorenz-equivalent model.

The intensity wave form represented in Fig. 1(a) is highly irregular and is apparently chaotic. This behavior is consistent with previous studies of pulsations in homogeneously broadened lasers under similar conditions. However, it is found that this behavior may change substantially for noninfinite values of the normalized center frequency $z_{0}$, or equivalently for values of the homogeneous linewidth larger than zero $\left(\gamma=\pi \Delta v_{h}\right)$. The waveform segment shown in Fig. 1(b) is obtained at the value $z_{0}=100$. While the pulsations in this case are still irregular, the behavior is somewhat more subdued than in Fig. 1(a). In Fig. 1(c) the frequency is $z_{0}=50$, and in this case the irregularity is gone, as the wave form repeats after every four pulsations. A more peaceful version of the period-four pulsations is obtained at $z_{0}=20$, as shown in Fig. 1(d). With $z_{0}=10$, as in Fig. 1(e), the period-two wave form consists of similar pulses simply alternating in height; while with $z_{0}=5$, as in Fig. 1 (f), the pulses are all essentially identical.

The conclusion to be drawn from Fig. 1 is that, at least for this general range of parameters, a more accurate model which avoids the derivative approximation based on the assumption of slowly varying amplitudes tends to be somewhat more stable than the familiar model which incorporates this approximation. Avoiding the approximation may be understood to allow the magnetic field to drag along behind the electric field, since the magnetic field experiences neither the gain (electric dipole transition) nor the loss (electric conductivity) encountered by the electric field. This dragging of the magnetic field seems to have the effect of a viscous force tending to stabilize the oscillations. The actual instability thresholds are also altered. In principle, the type 1 or perturbation instability threshold can be obtained from a solution of the linearized equations, but in practice direct numerical solutions may be the simplest way to derive the stability boundaries.

The relationship between the electric and magnetic fields can be explored in more detail by examining the instantaneous energy densities and frequencies of these fields. Figure 2(a) shows the instantaneous energy density $U_{m}(t)$ and frequency shift $\Delta \omega_{m}(t)=\omega_{m}(t)-\omega_{0}$ associated with the magnetic field for a spontaneously pulsing laser under the same conditions as discussed previously and with the normalized frequency $z_{0}=5$. A comparison of the energy density curve of Fig. 2(a) with the intensity curve of Fig. 1(f) shows that the peak value of the magnetic energy density is slightly less than the peak value of the intensity. The frequency units in Fig. 2 correspond to the normalized time units of $\gamma_{c}^{-1}$. It is clear from the figure that the average value of the instantaneous frequency shift is about $\Delta \omega_{m}=-0.17$. This means that the average frequency of a laser having its empty cavity frequency at the center of the atomic resonance will be below the resonance frequency by $0.17 \gamma_{c}$.

The corresponding results for the electric field are
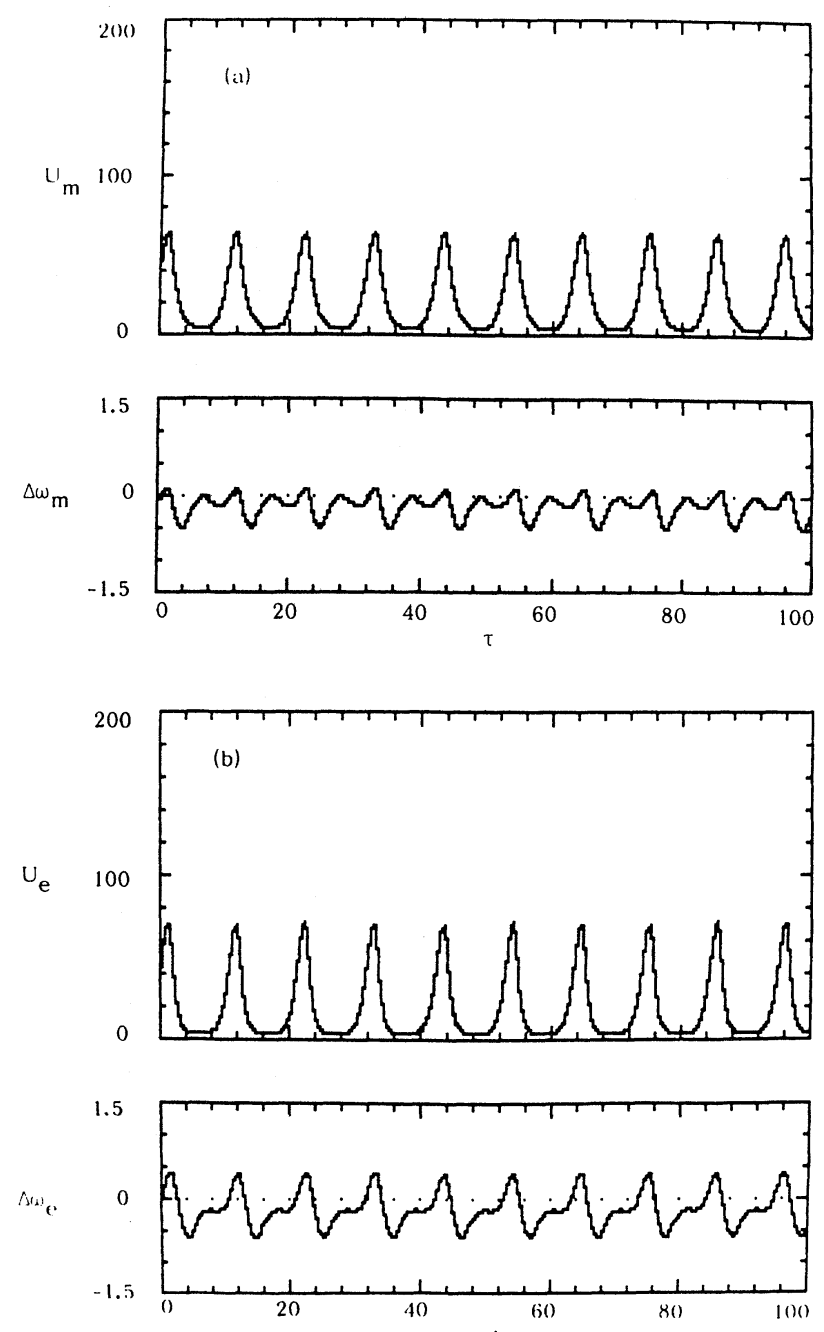

FIG. 2. Spontaneous pulsation energy density and instantaneous frequency shift wave forms for the same laser as Fig. 1(f). (a) shows the energy and frequency of the magnetic field, and (b) shows the same quantities for the electric field. The peak electric energy density is slightly higher and the peak magnetic energy slightly lower than the corresponding peak intensity. Both fields experience an average downward shift in frequency from line center. 
shown in Fig. 2(b). The peak value of the electric energy density is slightly larger than the peak of either the magnetic energy density or the intensity. This is to be expected since only the electric field experiences the electric dipole gain. It is also not surprising that the electric field exhibits wider frequency excursions than the magnetic field. The average values of the electric-field and magnetic-field frequencies are equal.

An alternative way to examine the relationship of the electric and magnetic fields is to plot their energy densities against each other. Figure 3 contains a plot of the magnetic energy density given by Eq. (68) versus the electric energy density given by Eq. (67). If the electric and magnetic energies had been equal, as implied in most analyses, this plot would be a simple straight line along the diagonal of the graph. In fact, however, for finite values of $z_{0}$, this plot becomes a two-dimensional trajectory, which in this instance resembles a distorted figure eight. The curve is traversed by the energy densities in the direction indicated by the arrows.

One of the consequences of having a more accurate model for the dynamics of a laser is that one must also be more specific in describing the cavity losses. When setting up the field equations for most laser models, it is conventional to simply generalize the conductivity losses to include all of the other losses that might occur due to scattering, absorption, mirror transmission, etc., and that same idea was employed in the above derivations. With the usual derivative approximation, there is no loss of accuracy or generality resulting from this approach. However, for the more accurate treatment being considered here, it may be important to distinguish between the loss rates for the electric and magnetic fields. One can, for example, envision circumstances in which there are equal losses for the electric and magnetic fields. In that case Eqs. (55) to (58) should be replaced by

$$
\begin{aligned}
\frac{d A_{r}(t)}{d t}=-\gamma_{c}[ & A_{r}(t)+\delta z_{0}\left[A_{i}(t)-B_{i}(t)\right] \\
& \left.-\frac{2}{z_{0}}\left[P_{r}(t)-A_{i}(t) D(t)\right]+2 P_{i}(t)\right],
\end{aligned}
$$

$$
\begin{aligned}
\frac{d A_{i}(t)}{d t}=-\gamma_{c}[ & A_{i}(t)-\delta z_{0}\left[A_{r}(t)-B_{r}(t)\right] \\
& \left.-\frac{2}{z_{0}}\left[P_{i}(t)+A_{r}(t) D(t)\right]-2 P_{r}(t)\right],
\end{aligned}
$$

$$
\begin{aligned}
& \frac{d B_{r}(t)}{d t}=-\gamma_{c}\left\{B_{r}(t)+\delta z_{0}\left[B_{i}(t)-A_{i}(t)\right]\right\}, \\
& \frac{d B_{i}(t)}{d t}=-\gamma_{c}\left\{B_{i}(t)-\delta z_{0}\left[B_{r}(t)-A_{r}(t)\right]\right\} .
\end{aligned}
$$

One readily finds that in the limit of large $z_{0}$ the set of equations given as Eqs. (71) to (74) reduces to Eqs. (51) and (52). However, for finite values of $z_{0}$, the predictions

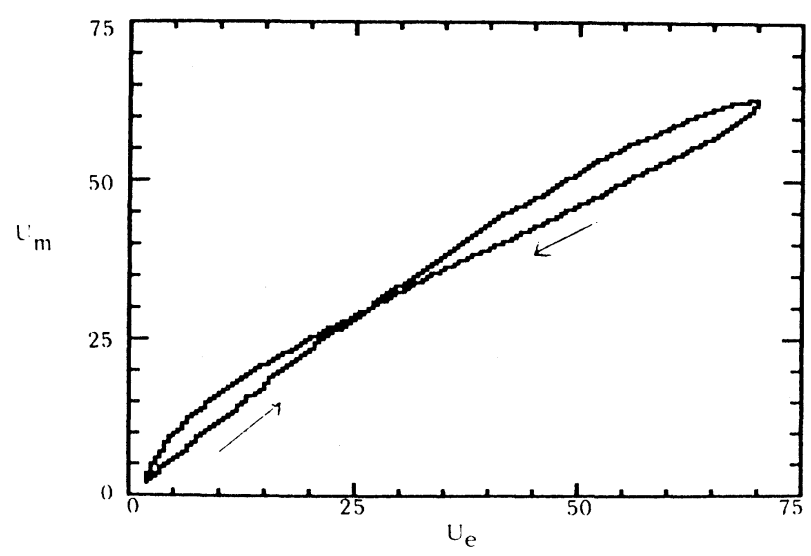

FIG. 3. Magnetic energy vs electric energy in the spontaneously pulsing laser represented previously in Figs. 1(f) and 2. The path in the figure is traversed periodically in figure-eight fashion in the direction shown by the arrows.
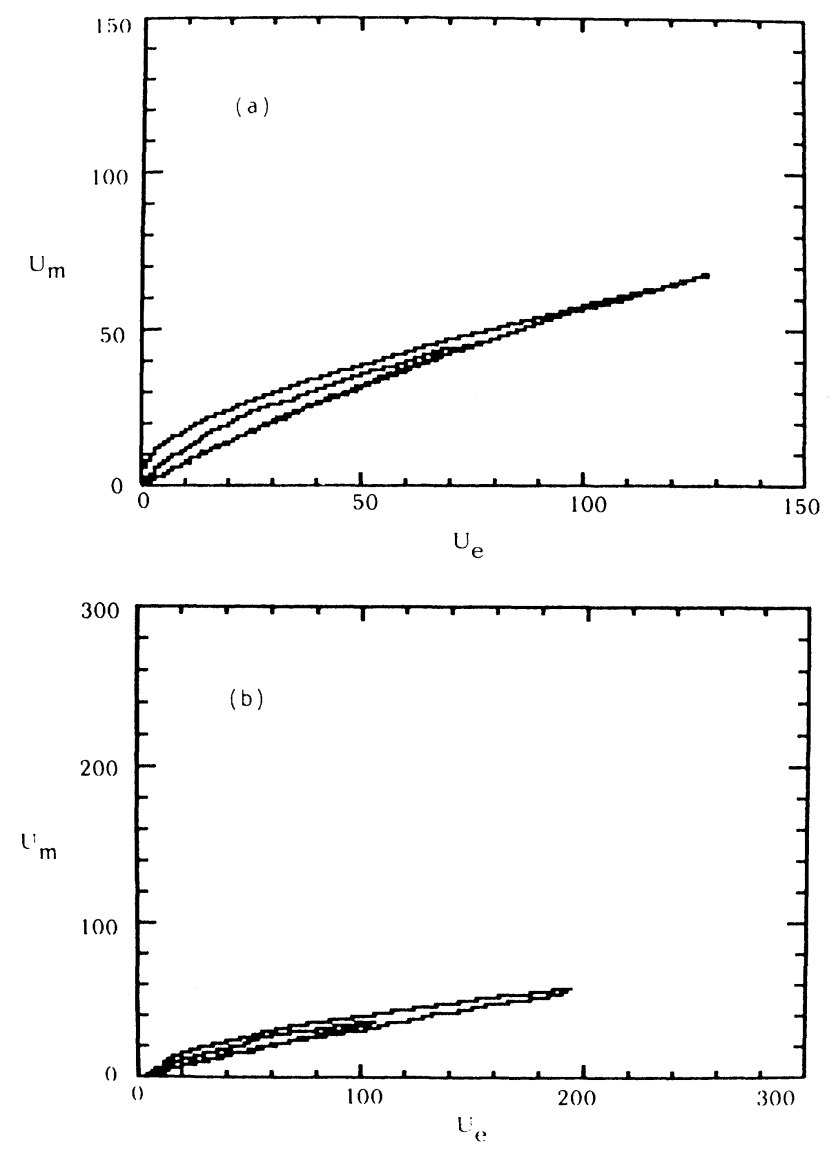

FIG. 4. Magnetic energy vs electric energy in a spontaneously pulsing laser in which (a) electric and magnetic losses are equal and (b) electric losses vanish. The wave forms corresponding to both (a) and (b) consist of alternating pulse heights, and the associated large and small loops in the figures are all traversed in the counterclockwise direction. It is clear that the energy advantage of the electric field in comparison to the magnetic field increases as the losses are transferred to the magnetic field. 
of the model using Eqs. (71) to (74) may be significantly different from those using Eqs. (55) to (58). In particular, for this change of decay rates, the plot of $U_{m}$ versus $U_{e}$ given in Fig. 3 must be replaced by the plot in Fig. 4(a). In this revised situation the pulses are again alternating in amplitude, and it is this alternation that leads to the double loop in the figure. Both loops are traversed in the counterclockwise direction. It is reasonable that when only the electric field has gain and both fields have loss, the electric-field amplitude tends to reach larger values than the magnetic-field amplitude.

For completeness one may also consider the extreme case in which all of the losses are associated with the magnetic field. In this limit Eqs. (71) to (74) are replaced by

$$
\begin{aligned}
\frac{d A_{r}(t)}{d t}=-\gamma_{c}[ & +\delta z_{0}\left[A_{i}(t)-B_{i}(t)\right] \\
& \left.-\frac{2}{z_{0}}\left[P_{r}(t)-A_{i}(t) D(t)\right]+2 P_{i}(t)\right],
\end{aligned}
$$

$$
\begin{aligned}
\frac{d A_{i}(t)}{d t}=-\gamma_{c}[ & -\delta z_{0}\left[A_{r}(t)-B_{r}(t)\right] \\
& \left.-\frac{2}{z_{0}}\left[P_{i}(t)+A_{r}(t) D(t)\right]-2 P_{r}(t)\right],
\end{aligned}
$$

$$
\begin{aligned}
& \frac{d B_{r}(t)}{d t}=-\gamma_{c}\left\{2 B_{r}(t)+\delta z_{0}\left[B_{i}(t)-A_{i}(t)\right]\right\}, \\
& \frac{d B_{i}(t)}{d t}=-\gamma_{c}\left\{2 B_{i}(t)-\delta z_{0}\left[B_{r}(t)-A_{r}(t)\right]\right\} .
\end{aligned}
$$

Now the plot of $U_{m}$ versus $U_{e}$ given in Fig 4(a) is replaced by the plot in Fig. 4(b). It is clear that this change favors even further the electric field in comparison to the magnetic field.

\section{GIANT PULSES AND RELAXATION OSCILLATIONS}

Section IV has indicated some of the ways in which the derivative approximation in the field equations affects the spontaneous pulsation predictions of a semiclassical laser model. It is important to note that this approximation is also made (or implied) in studies of such laser transients as giant pulses and relaxation oscillations, and these transient effects are often of more practical importance than possible instability phenomena. A giant pulse is the large intensity burst that occurs when a laser has its gain switched to a high value (gain switched) or its loss switched to a low value (loss switched, $Q$ switched) in a time that is short compared to the rise time of the optical pulse. If the pumping is maintained after the gain pulse, then that pulse is followed by a damped train of relaxation oscillations leading ultimately to steady-state operation.
Spontaneous pulsations can be eliminated from the model discussed above by simply increasing the value of $\delta=\gamma / \gamma_{c}$. Figure 5 includes plots of the initial transients for a prepumped laser with $\delta=2$ and a small signal injected at time $\tau=0$. The other parameters of the laser are $\rho=0.2$ and $r=20$ as before, and the computations are again based on Eqs. (48) to (50) and (55) to (58). The intensity is plotted for several values of the normalized center frequency $z_{0}$. The curve in Fig. 5(a) is obtained for the limit $z_{0}=\infty$. In this case the output consists of an initial pulse with a peak intensity of about $I_{p} \approx 118$, and since pumping is continued, there is also a damped train of relaxation oscillations leading to the steady-state intensity $I_{s}=r-1=19$. As one could anticipate from the results given previously, the smaller values of $z_{0}$ used in Figs. 5(b) and 5(c) lead to a slightly smaller giant pulse and more strongly damped relaxation oscillations. The
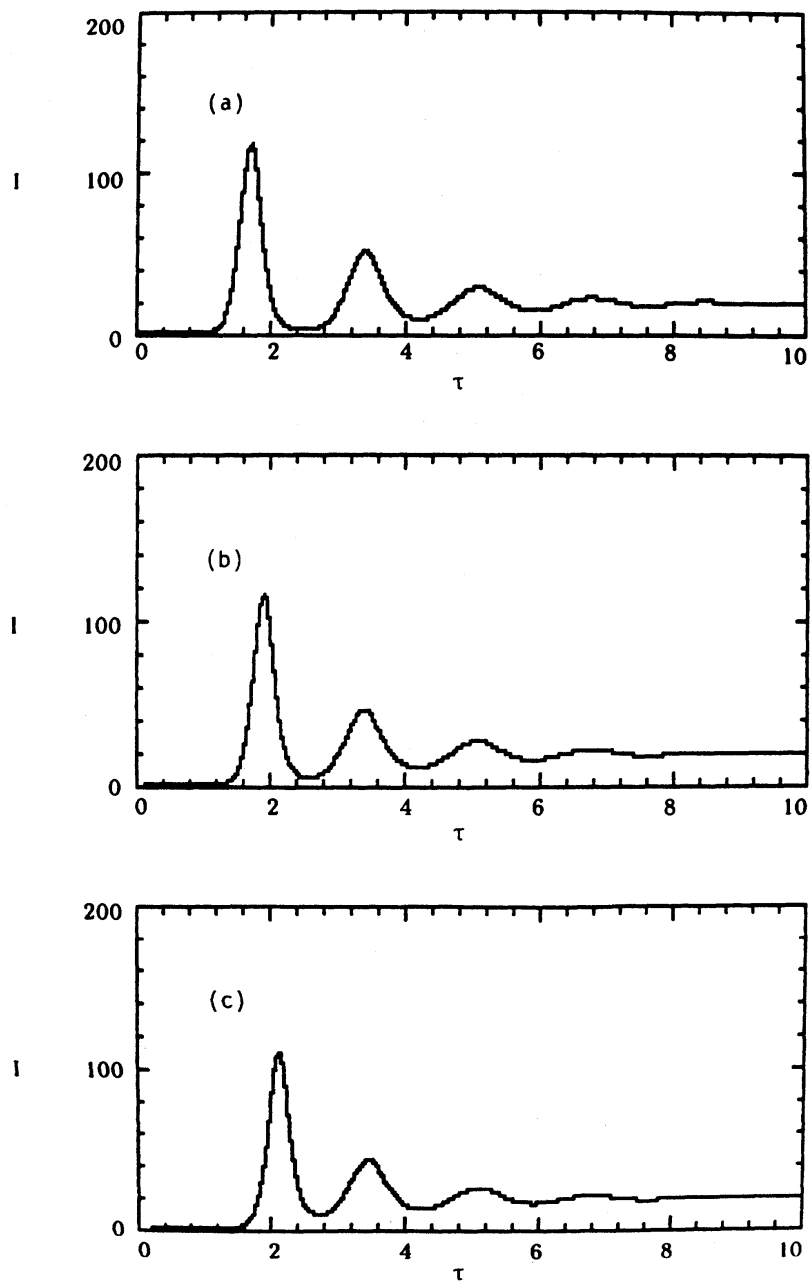

FIG. 5. Giant pulsations and relaxation oscillations for a prepumped and loss-switched laser governed by the parameters $\delta=2, \rho=0.2$, and $r=20$. The normalized center frequencies $z_{0}$ for these plots are (a) $\infty$, (b) 10, and (c) 5 . The smaller values of $z_{0}$ lead to slightly weaker pulsations. 
instantaneous frequency shift is zero for all time for $z_{0}=\infty$ but oscillates and damps to zero for the conditions of Figs. 5(b) and 5(c).

\section{CONCLUSION}

In studies of laser dynamics it is usual to incorporate a derivative approximation based on the assumption that the amplitude of the electric field varies slowly compared to an optical cycle. The purpose of this study has been to develop models of laser dynamics which do not make this approximation. It is found that in high-gain and largebandwidth systems, the derivative approximation can cause significant errors in predicted laser behavior. At least for some parameter combinations, avoidance of the derivative approximation leads to more strongly damped pulsations than would otherwise be expected. Examples of systems where these corrections might be significant would include the very high gain dye and quantum well laser systems that are likely to find many practical applications. Even where the quantitative implications of avoiding the approximation are not large, a formal awareness of these implications will lead to greater confidence in any theoretical predictions. Also, it should be emphasized that, while the discussion here has been limited to the simplest possible time-dependent laser configurations, related corrections would also be expected in more complicated multimode systems.

Note added in proof. Since the acceptance of this manuscript we have become aware of a related study by Li and Mandel. ${ }^{15}$

\section{ACKNOWLEDGMENTS}

This work was supported in part by the National Science Foundation and by Tektronix, Inc. The author also expresses his appreciation to W. J. Sandle, R. J. Ballagh, D. M. Warrington, and other members of the Department of Physics at the University of Otago, Dunedin, New Zealand, for valuable discussions and hospitality during his sabbatical visit.
"Permanent address: Department of Electrical Engineering, Portland State University, P.O. Box 751, Portland, OR 97207-0751.

${ }^{1}$ L. W. Casperson and A. Yariv, IEEE J. Quantum Electron. QE-8, 69 (1972).

${ }^{2}$ L. W. Casperson, IEEE J. Quantum Electron. QE-14, 756 (1978).

${ }^{3}$ L. W. Casperson, J. Opt. Soc. Am. B 2, 62 (1985).

${ }^{4}$ L. W. Casperson, Spontaneous Pulsation in Lasers, Vol. 182 of Lecture Notes in Physics (Springer-Verlag, Berlin, 1983), pp. 88-106.

5J. C. Englund, R. R. Snapp, and W. C. Schieve, Prog. Opt. 21, 355 (1984).

${ }^{6}$ J. R. Ackerhalt, P. W. Milonni, and M.-L. Shih, Phys. Rep.
128, 205 (1985).

${ }^{7}$ R. G. Harrison and D. J. Biswas, Prog. Quantum Electron. 10, 147 (1985)

${ }^{8}$ W. J. Firth, in Chaos, edited by A. V. Holden (Princeton University Press, Princeton, New Jersey, 1986), pp. 135-157.

${ }^{9}$ N. B. Abraham, P. Mandel, and L. M. Narducci, Prog. Opt. 25, 1 (1988).

${ }^{10}$ C. O. Weiss, Opt. Quantum Electron. 20, 1 (1988).

${ }^{11}$ L. W. Casperson, Phys. Rev. A 42, 6721 (1990).

${ }^{12}$ L. W. Casperson, J. Opt. Soc. Am. B 2, 73 (1985).

${ }^{13}$ H. Haken, Phys. Lett. 53A, 77 (1975).

${ }^{14}$ L. W. Casperson, J. Opt. Soc. Am. B 5, 970 (1988).

${ }^{15}$ R.-D. Li and P. Mandel, Opt. Commun. 75, 72 (1990). 